\title{
The Effect of Inter-Repetition Rest Duration on Kinematic of Snatch
}

\author{
${ }^{1}$ Kevin Tan ${ }^{\circledR},{ }^{1}$ Nur Ikhwan bin Mohamad ${ }^{\circledR},{ }^{1}$ Ali Md Nadzalan ${ }^{(\mathbb{D} *}$ \\ ${ }^{1}$ Faculty of Sports Science and Coaching, Universiti Pendidikan Sultan Idris, Perak, Malaysia.
}

Submitted 07 December 2020; Accepted in final form 12 February 2021.

\begin{abstract}
Background. Controlling and manipulating inter-repetition rest (IRR) could develop diverse levels of fatigue and manifest changes to lifting mechanics. Objectives. This study's objective was to examine the effect of IRR on kinematic of snatch lifting during multiple set exercise protocol. Methods. Fifteen male $(n=15)$ athletes participated in this study (age $=21.0 \pm 1.41$ years; body weight $=60.82 \pm 2.45 \mathrm{~kg}$; height $=165.70 \pm 10.88 \mathrm{~cm}$; snatch one-repetition maximum $(1 \mathrm{RM}) /$ body mass $=0.73 \pm 0.117)$. Session 1 consisted of anthropometric and $1 \mathrm{RM}$ determination. Sessions 2-4 involved subjects performing three sets x 5 repetitions of 85\% $1 \mathrm{RM}$ with 10,30, or 50 seconds of IRR implemented randomly. Ankle, knee, and hip joint velocity and barbell velocity (BV) were obtained during each protocol using VICON Motion Analysis (100Hz). Results. Repeated measure ANOVA showed significant differences found in ankle, knee, and hip joint velocity. The barbell velocity showed a significant effect between IRR, $(\mathrm{F}(2,28)=22.831, \mathrm{P}<$ 0.05). Repeated measure Manova showed a significant effect of IRR on the maintenance of kinematics variables across repetition. Barbell velocity showed a significant effect of IRR on the maintenance of velocity across repetition $(\mathrm{P}=$ 0.029). Conclusion. Due to the differences found between IRR protocols, the implementation of IRR may increase or decrease kinematic output due to the different levels of fatigue. The implementation of IRR can help to maintain kinematic variables, which often are affected by neuromuscular fatigue occurred with continuous repetition.
\end{abstract}

KEYWORDS: Weightlifting, Joint Kinematics, IRR, Snatch, Biomechanics.

\section{INTRODUCTION}

The introduction of the weightlifting movement and its affiliate training regime has become somewhat crucial in an athlete's training program. A viral weight training workout that is widely practiced in the snatch lift. It is an exercise in which the athletes would lift the heaviest weight as possible in snatch exercise (1). The snatch is known as an explosive exercise and overall body exercise.

The capability to demonstrate an optimum technique during snatch is dependent on the level of fatigue. Due to this factor, the ability to maintain and retain good technique form over multiple repetitions would be of interest to coaches and athletes. Fatigue will occur in the human body as it is how the body physiology works. The best we could do is to slow down the effect of fatigue on performance output in any sports because fatigue not only impaired performance but also the technique (2). To backed the claim that fatigue would be detrimental to the performance and kinematic of athletes, Hardee et al. (3), Hardee et al. (4), Oliver et al. (5), and ValverdeEsteve et al. (6) has shown that with increasing intensities or loads during weightlifting training by either using a barbell or dumbbell exercise could lead to increase in fatigue level and would negatively affect the technique, rate of perceived effort and power output.

*. Corresponding Author:

Ali Bin Md Nadzalan, Ph.D

E-mail: ali.nadzalan@fsskj.upsi.edu.my 
Besides the overall motor control, joints sequencing timing, barbell trajectory, and snatch exercise experience, the joints movement is also one of the key contributors in producing an optimum snatch movement. The ankle, knee, and hip joint specifically play a crucial role in the snatch process. The increase in performance depends on the improvement of proper technique and training methods (7). Determining the effects of IRR on the kinematics profiles is indispensable to find the practical technical factors of successful lifts (8). If one of these joints failed to function correctly, it could poorly reflect the snatch lift movement. Injury may occur due to fatigue (9, 10). The barbell velocity is also considered one factor in determining an optimum snatch lift (11).

To preserve and maximize the performance effect an athlete produced during training, interrepetition rest (IRR) protocol has been given to athletes to regress and maintain performance variables such as power, force, and velocity $(3,5$, $12,13)$. IRR is defined as rest taken in between each repetition with a duration from small to moderate in the given volume of training. The selection of an appropriate rest interval is essential to maintain a high velocity and performance output throughout the repetition and set. However, biomechanically, little to nonresearch has been done to prove this at the joint level. The main reason joint velocity and barbell velocity was chosen is the rising importance of velocity monitoring in training (11). By monitoring the velocity output, we can consider fatigue in athletes during training with different protocols (14).

Research about inter repetition rest is still in its infancy, and many theories are surrounding inter-repetition rest training that is currently unsupported. The behavior of joint kinematic during snatch training with different interrepetition rest (IRR) has not been clearly established. Thus, the objective of the present study was to determine the effect of Interrepetition rest (IRR) with different duration (10s, the 30s, and 50s) on the joint angular velocity, barbell velocity and also to compare the capacity to maintain the joint rate and barbell velocity across the repetition and sets of snatch exercise given.

\section{MATERIALS AND METHODS}

Participants. Fifteen healthy male $(n=15)$, currently active athletes (sprinting, throwing, rugby, football, or futsal) who has been trained in weight training exercises (age: $21 \pm 1.41$ years, weight: $60.82 \pm 2.45 \mathrm{~kg}$, height: $165.70 \pm 10.88$ $\mathrm{cm}, 1 \mathrm{RM}$ of snatch relative to body mass: $0.73 \pm$ $0.117 \mathrm{~kg} \mathrm{~kg}^{-1}$ ) volunteered to participate in this study. Fifteen participants are chosen based on a previous study with a similar design $(4,15)$. The inclusion criteria for participation were: (a) an ability to lift at least $50 \%$ of their body weight during the snatch exercise; (b) at least six months of experience in a snatch training exercise; and (c) the non-use of drugs or dietary supplements that could affect physical performance. All participants were informed of the benefits and risks of the investigation before signing an informed consent form by the Sultan Idris Education University Institutional Review Board and The Code of Ethics of the World Medical Association (Declaration of Helsinki).

Procedure. (Session 1) Each participant undertook four testing sessions, 72 hours apart. Session 1 was used for familiarization of equipment, descriptive data, and $1 \mathrm{RM}$ testing and collection. In Session 1, the participant's descriptive data and one-repetition maximum (1RM) were determined in the snatch exercise. 1RM was performed in the manner described by Baechle \& Earle. (16). Participants were instructed to complete between one and three repetitions per set and began their first set with the barbell only (20kg). Additional weight was added to the barbell each stage until the load reached approximately $60 \%$ of the estimated 1RM. After that, $5-10 \mathrm{~kg}$ was added to the bar until the weight reached about $90 \%$ of the estimated 1RM, where then only 2.5 or $5 \mathrm{~kg}$ was added to the barbell until the 1RM was determined. One repetition maximum was selected after participants missed two successive attempts at a given load. A portable stadiometer and digital scales (Model BSM370, InBody, Seoul, Korea) were used to measure the height $(\mathrm{cm})$ and body weight $(\mathrm{kg})$, respectively. A vernier caliper and a measuring tape was used to measure the anthropometric data needed for the Vicon software. This session was monitored by an expert from the strength and conditioning field.

(Session 2-4) All participants arrived at the laboratory 72 hours after completing Session 1 . In Session 2-4, after the warm-up session, each participant performed three sets of five repetition snatches with five minutes of rest given between groups for one of the three IRR protocol 
conditions in each session: $10 \mathrm{~s}$, the $30 \mathrm{~s}$, or $50 \mathrm{~s}$ IRR protocol each session until completed.

Reflective markers were then placed according to the Vicon plug-in gait default lower body marker set. Three reflective qualities at also placed at both end and middle of the barbell. Precisely, markers were placed on the left and right anterior superior iliac spine, left and right lateral thigh, left and right lateral epicondyle of the knee, left and right medial epicondyle of the knee (calibration only), left and right lateral tibia, left and right lateral malleolus, left and right medial malleolus, left and right second metatarsal, and left and right heel.

All participants performed the snatch using $85 \%$ of their 1RM loads, and all repetitions were executed as quickly as possible. IRR sets were performed in a counterbalanced order between participants. During each repetition, peak joint velocity was recorded for each phase of the snatch. The peak barbell velocity was also recorded using a VICON Nexus $(1,13)$ Real-time Motion Analysis system (Vicon Motion Systems Ltd UK, West Way, Oxford). Kinematics data were capture at $100 \mathrm{~Hz}$ using a motion capture system with a 6 Vicon T-10s camera (Vicon Motion Systems Ltd UK, West Way, Oxford) with markers placed bilaterally on the hips, knee, and ankle joint.

All phases of snatch observe in this study are as follows. The first pull phase is from the barbell lift-off until the first maximum knee extension (17). The transition phase is described as a movement from the first full knee extension until the early maximum knee flexion (17). The second pull phase happens from the first maximum knee flexion until the second maximum extension of the knee (17).

Data Processing. The joints kinematic is taken using the Vicon Nexus (Vicon Motion Systems Ltd UK, West Way, Oxford) system set at $100 \mathrm{~Hz}$. The raw data will then be processed using VICON Polygon (Vicon Motion Systems Ltd UK, West Way, Oxford). Kinematic data will be filtered using 4th order Butterworth filter with cut-off frequency at $6 \mathrm{~Hz}$. $(18,19)$.

Statistical Analysis. Results are presented as mean $( \pm)$. The hypotheses of normality and homogeneity of the variance were analyzed via Kolmogorov-Smirnov and Levene tests, respectively. A repeated measure analysis of variances (ANOVA) was used to compare the difference of joints velocity (AJV, KJV, HJV) and barbell velocity (BV) between different duration of IRR protocol on various phases of the snatch. When a significant interaction was found, posthoc tests with Bonferroni correction were used to localize the effect. Repeated measure Multivariate analysis of variances (MANOVA) was also used to compare the maintenance of kinematics variables between repetition one and repetition five on the peak values of the ankle, knee, and hip joint angular velocity and maximum barbell velocity with different IRR protocols. When a significant interaction was found, univariate ANOVA with Bonferroni correction tests was used to evaluate further the kinematic differences between the first repetition and fifth repetition for the maintenance of velocity objective. Statistical significance is accepted at an alpha level of $P \leq 0.05$. All statistical analyses will be performed using SPSS 25 (IBM, Inc., Chicago, IL).

\section{RESULTS}

Joint Angular Velocity. In the first pull phase, the ankle and hip show the fastest angular velocity in the 50s IRR protocol, while the knee shows a faster joint angular velocity in the 30s IRR protocol. However, there is no significant IRR effect on the maximum ankle joint velocity during this phase, $\mathrm{F}(2,28)=2.591, \mathrm{P}=0.093)$.

The knee joint velocity showed a significant effect between IRR, F $(1.181,16.538)=4.322, \mathrm{P}$ $=0.048$ ). A post hoc pairwise comparison using the Bonferroni correction was used to localize the effect. The knee joint velocity in the 30 s IRR protocol is significantly faster than the 50s IRR protocol $(4.78 \mathrm{rad} / \mathrm{s}$ vs. $4.24 \mathrm{rad} / \mathrm{s})(\mathrm{P}=.039)$. There is no significant effect of IRR protocol on the hip joint angular velocity. However, it can be seen in Table 1 that the mean peak hip joint velocity is faster in the 50s IRR protocol compared to the 10s and 30s IRR protocol.

During the transition (T1) phase in the snatch, the ankle, knee, and hip joint showed a faster angular velocity in the 30s IRR protocol than other protocols. There is a significant effect between IRR, F $(2,28)=7.355, \mathrm{P}=0.003)$ for ankle joint velocity. A post hoc pairwise comparison was used to localize the effect. The 10 s IRR protocol is significantly faster in joint velocity compared to the 50s IRR protocol, (2.02 $\mathrm{rad} / \mathrm{s}$ vs $1.69 \mathrm{rad} / \mathrm{s}$, respectively), ( $\mathrm{p}=.028)$. Furthermore, the ankle joint velocity also is significantly faster in the 30s IRR protocol 
compared to the 50s IRR protocol group $(2.28 \mathrm{~m} / \mathrm{s}$ vs $1.69 \mathrm{rad} / \mathrm{s}),(\mathrm{P}=0.011)$.

As for the knee joint velocity, there is a significant effect between IRR, $\mathrm{F}(2,28)=8.520$, $\mathrm{P}=0.001$ ). A post hoc pairwise comparison was used to localize the impact. The 30s IRR protocol is significantly faster than the knee joint velocity of the 50s IRR protocol in this phase $(2.87 \mathrm{rad} / \mathrm{s}$ vs. $2.00 \mathrm{rad} / \mathrm{s}),(\mathrm{P}=0.007)$.

On the other hand, the hip joint also showed a significant effect of IRR, F $(2,28)=9.767, \mathrm{P}=$ 0.001). A post hoc pairwise comparison showed that the hip joint in 30s IRR moves faster than the 10s IRR protocol significantly (4.91 rad/s vs. 4.28 $\mathrm{rad} / \mathrm{s}$, respectively), $(\mathrm{P}=0.019)$. This is also observed when comparing the 30s IRR protocol with the $50 \mathrm{~s}$ IRR protocol $(4.91 \mathrm{rad} / \mathrm{s}$ vs. 4.30 $\mathrm{rad} / \mathrm{s}),(\mathrm{P}=0.005)$. The hip joint velocity in the $30 \mathrm{~s}$ IRR protocol is significantly faster than the hip joint velocity of the 50s IRR protocol in this phase.

In the second pull (SP) phase, there is a significant effect between IRR, F $(2,28)=9.892$, $\mathrm{P}=0.001$ ) for hip joint velocity in this phase. A post hoc pairwise comparison was used to localize the effect. The hip joint angular velocity showed a significant difference between the $10 \mathrm{~s}$ IRR protocol and the 30s IRR protocol. The hip joint in 30s IRR moves faster than the 10s IRR protocol significantly $(6.30 \mathrm{rad} / \mathrm{s}$ vs. $5.49 \mathrm{rad} / \mathrm{s}$, respectively $),(P=0.001)$. However, the ankle and the knee joint velocity didn't show a significant difference between the three types of IRR protocol tested in this study $(\mathrm{P}=0.391$ and $\mathrm{P}$ $=0.065$, respectively).

Table 1. Angular Velocity of the Ankle, Knee, and Hip Joints During the First Pull, Transition, and Second Pull. Data Are Means $( \pm$ SD)

\begin{tabular}{|c|c|c|c|}
\hline \multirow{2}{*}{ Variables } & 10s IRR & 30 IRR & 50 IRR \\
\hline & Means $( \pm$ SD $)$ & Means( $( \pm$ SD) & Means( $( \pm$ SD) \\
\hline \multicolumn{4}{|l|}{ First, pull } \\
\hline Maximum ankle (plantar-flex) velocity $(\mathrm{rad} / \mathrm{s})$ & $1.82(0.22)$ & $1.99(0.27)$ & $2.02(0.39)$ \\
\hline Maximum knee extension velocity (rad/s) & $4.17(0.95)$ & $4.78(1.88)$ & $4.25(1.33)$ \\
\hline Maximum hip extension velocity $(\mathrm{rad} / \mathrm{s})$ & $2.76(0.44)$ & $2.89(0.62)$ & $2.91(0.47)$ \\
\hline \multicolumn{4}{|l|}{ Transition } \\
\hline Maximum ankle (plantar-flex) velocity $(\mathrm{rad} / \mathrm{s})$ & $2.02(0.44)$ & $2.29(0.71)$ & $1.69(0.50)$ \\
\hline Maximum knee flexion velocity $(\mathrm{rad} / \mathrm{s})$ & $2.28(0.50)$ & $2.87(1.08)$ & $2.01(0.56)$ \\
\hline Maximum hip extension velocity ( $\mathrm{rad} / \mathrm{s})$ & $4.28(0.49)$ & $4.92(0.60)$ & $4.31(0.49)$ \\
\hline \multicolumn{4}{|l|}{ Second pull } \\
\hline Maximum ankle (plantar-flex) velocity $(\mathrm{rad} / \mathrm{s})$ & $6.79(1.75)$ & $7.28(0.71)$ & $6.96(2.21)$ \\
\hline Maximum knee extension velocity $(\mathrm{rad} / \mathrm{s})$ & $7.64(2.72)$ & $7.90(2.50)$ & $7.21(3.44)$ \\
\hline Maximum hip extension velocity ( $\mathrm{rad} / \mathrm{s})$ & $5.49(0.78)$ & $6.31(1.15)$ & $5.79(1.26)$ \\
\hline
\end{tabular}

IRR: Inter-Repetition Rest.

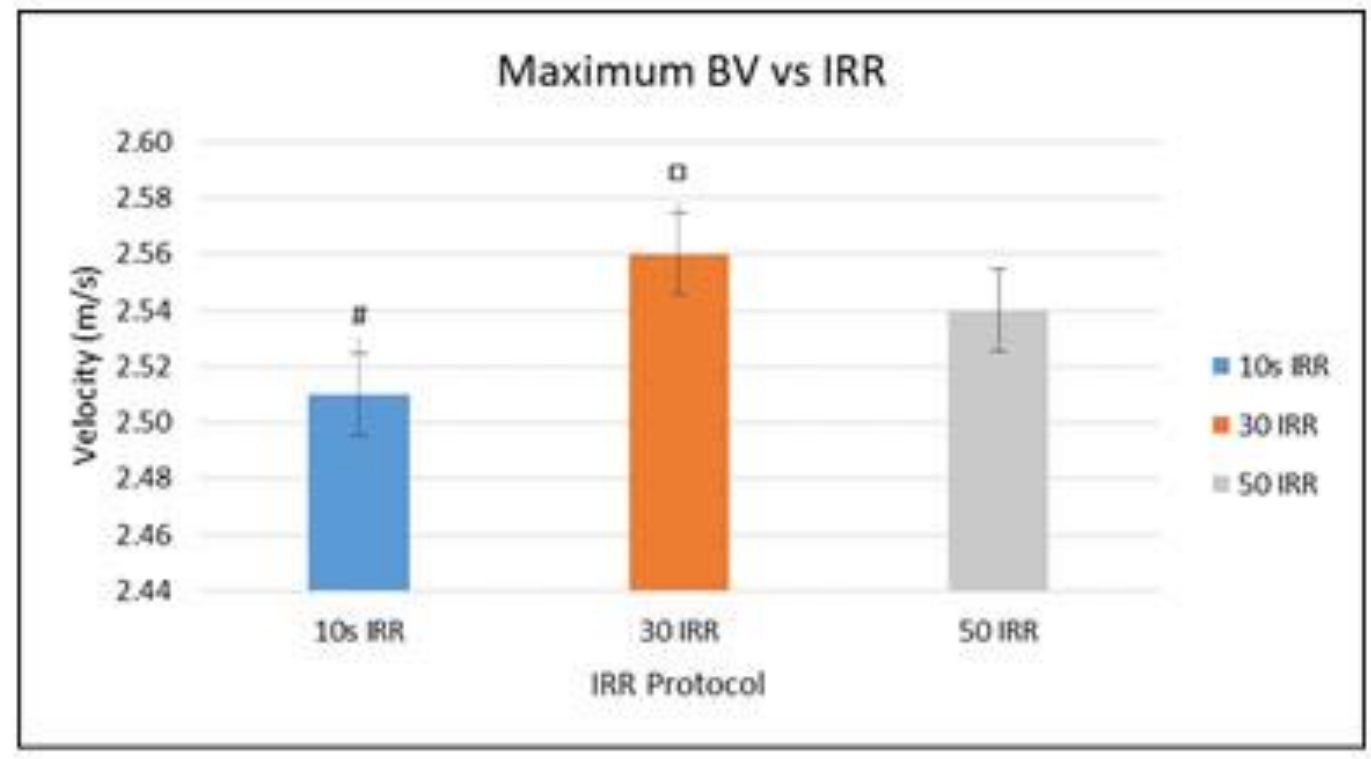

Figure 1. Maximu Barbell Velocity (m/s) vs IRR. \#.IRR significantly different from 30s and 50s. ${ }^{\square}$.IRR significantly different from 50s. $* / \square(\mathrm{P}<\mathbf{0 . 0 5})$. 
Barbell Velocity. The data presented shows the mean for the maximum barbell velocity during the full snatch across three different interrepetition rest duration protocols. The mean peak barbell velocity is the fastest in the 30 s IRR protocol than the 10s and 50s IRR protocol. A repeated-measures ANOVA determined that the peak barbell velocity during snatch differed significantly across three IRR duration protocol, $(\mathrm{F}(2,28)=22.831, \mathrm{P}<0.05)$. A post hoc pairwise comparison using the Bonferroni correction was applied to localize the effect. The 30s IRR is significantly faster than the 10s IRR protocol $(2.70 \mathrm{~m} / \mathrm{s}$ vs. $2.36 \mathrm{~m} / \mathrm{s}$, respectively), $(\mathrm{P}<0.05)$. The 50s IRR protocol also has a significantly faster barbell velocity than the 10s IRR protocol $(2.55 \mathrm{~m} / \mathrm{s}$ vs. $2.36 \mathrm{~m} / \mathrm{s}$, respectively) $(\mathrm{P}=.009)$. The 30s IRR protocol also portrays a significantly faster BV than the 50s IRR protocol BV $(2.70 \mathrm{~m} / \mathrm{s}$ vs. $2.55 \mathrm{~m} / \mathrm{s})(\mathrm{P}=.021)$.

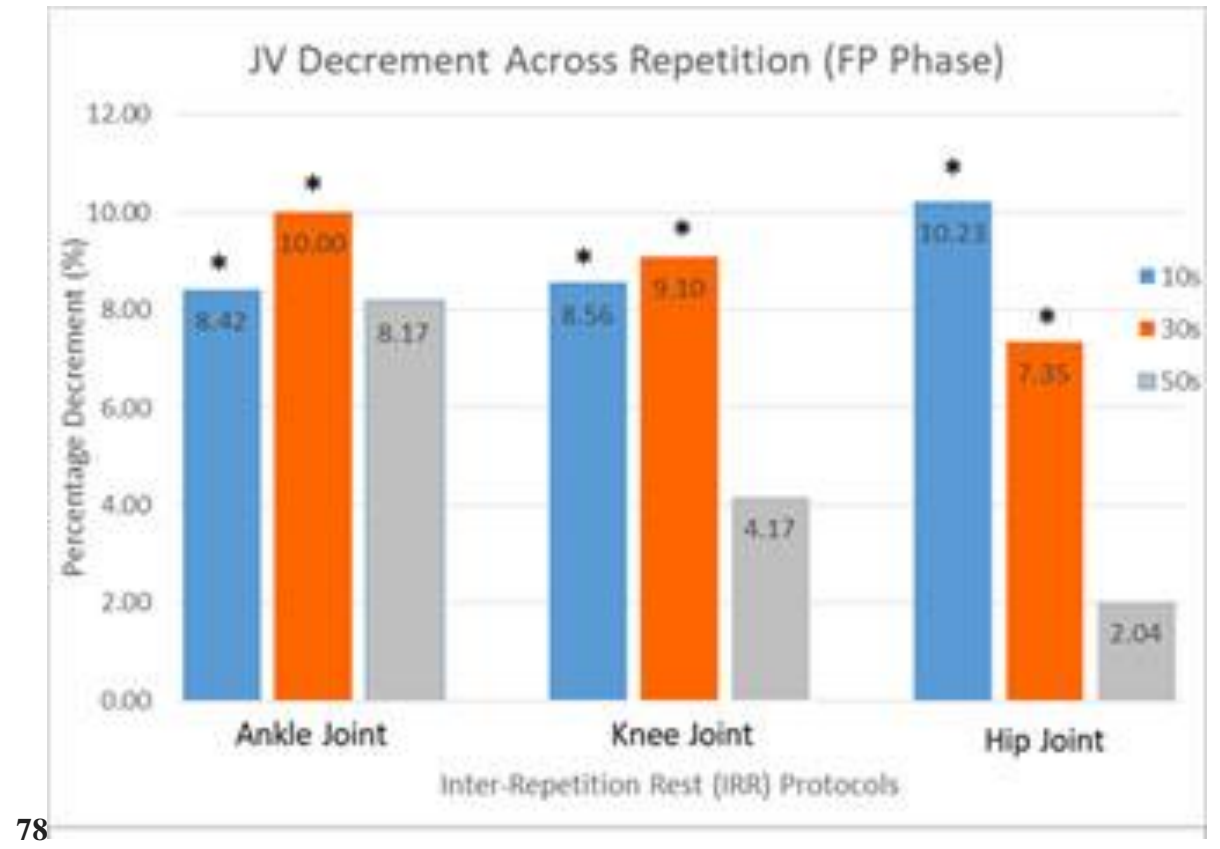

Figure 2. Joint Velocity Decrement (\%) During First Pull (FP) Phase Across Repetition. *, Significant drop in joint velocity during $5^{\text {th }}$ repetition $(\mathrm{P}<0.05)$.

Joint Velocity Maintenance. Figure 2 shows the result of the joint angle maintenance across repetition with the implementation of different IRR types (10s, the 30s, and 50s) during the first pull (FP) phase in the snatch. A repeated measure MANOVA test was conducted to test the intervention effect on AJV maintenance across repetition during the FP phase. There was a statistically significant difference between repetitions based on the type of IRR treatment given, $\mathrm{F}(3,12)=4.599, \mathrm{P}<0.023$; Wilk's $\Lambda=$ 0.465 , partial $\eta^{2}=0.535$, indicating a difference between R1 and R5 of AJV between the type of IRR treatment. Univariate ANOVA with Bonferroni Correction tests further showed that there was a significant difference between $\mathrm{R} 1$ and R5 for 10s IRR, $\mathrm{F}(1,14)=7.998, \mathrm{P}=0.013$, and 30s IRR, $\mathrm{F}(1,14)=4.654, \mathrm{P}=0.049$. There is no significant velocity drop between R1 and R5 for the 50s IRR protocol $(\mathrm{P}=0.109)$.
There was a statistically significant difference between repetitions based on the type of IRR treatment given, $\mathrm{F}(3,12)=7.458, \mathrm{P}<0.004$; Wilk's $\Lambda=0.349$, partial $\eta^{2}=0.651$. The Univariate ANOVA with Bonferroni Correction tests showed there was a significant difference between R1 and R5 for 10s IRR, F $(1,14)=$ 15.520, $\mathrm{P}=0.001$, and 30s IRR, $\mathrm{F}(1,14)=8.647$, $\mathrm{P}=0.011$. There is a significant drop in terms of KJV in these two IRR treatment group. However, the 50s IRR treatment group showed no significant difference between $\mathrm{R} 1$ and $\mathrm{R} 5(\mathrm{P}=0.302)$.

For the hip joint velocity maintenance, there was a statistically significant difference between repetitions based on the type of IRR treatment given, $\mathrm{F}(3,12)=6.167, \mathrm{P}<.009$; Wilk's $\Lambda=$ 0.393, partial $\eta^{2}=0.607$, indicating there is a difference between repetition one and repetition 5 of HJV with a different type of IRR treatment. The Univariate ANOVA with Bonferroni correction tests further showed there was a 
significant difference between $\mathrm{R} 1$ and $\mathrm{R} 5$ for $10 \mathrm{~s}$ IRR, $\mathrm{F}(1,14)=11.877, \mathrm{P}=0.004$, and 30s IRR, $\mathrm{F}(1,14)=6.641, \mathrm{P}=0.022$, showing that there is a significant drop in terms of HJV in this two IRR treatment group during FP phase. However, the 50s IRR treatment group showed no significant reduction in velocity between $\mathrm{R} 1$ and $\mathrm{R} 5(\mathrm{P}=$ 0.642 ).

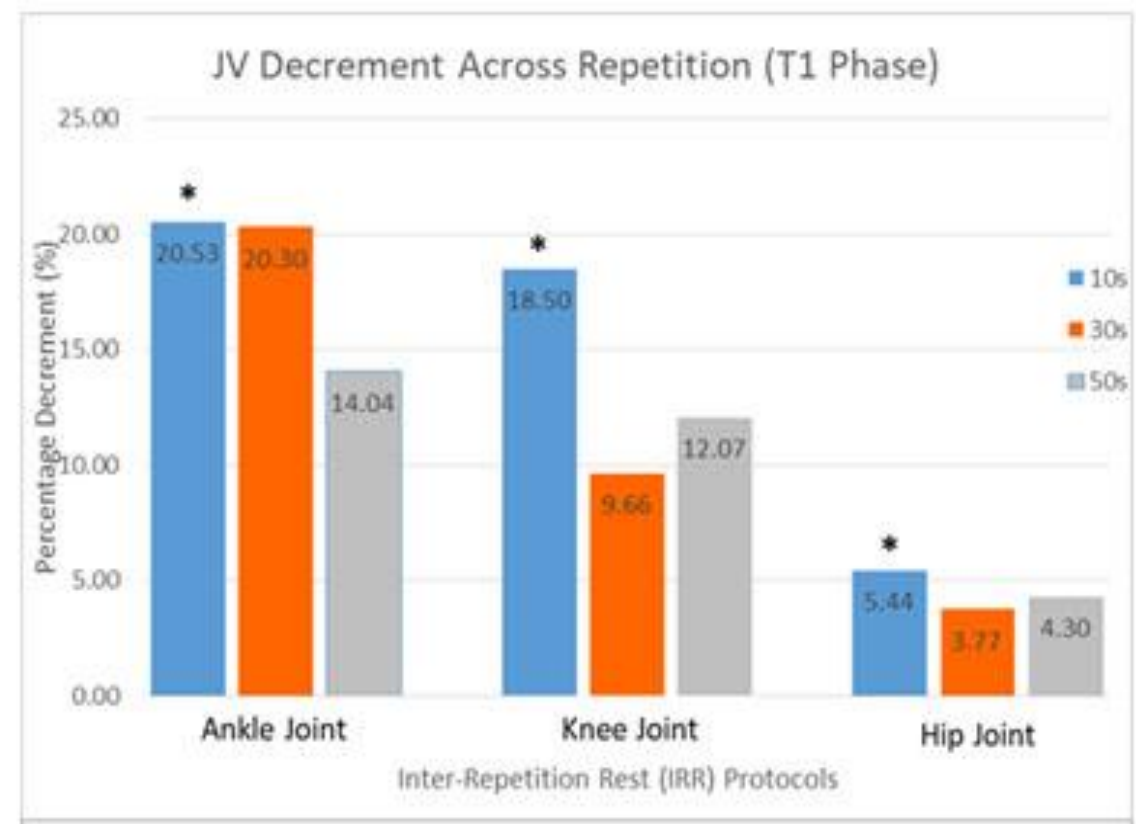

Figure 3. Joint Velocity Decrement (\%) During Transition (T1) Phase Across Repetitin. * Significant drop in jont velocity during $5^{\text {th }}$ repetition $(P<0.05)$.

Figure 3 present the joint angle velocity maintenance across repetition for the transition (T1) phase during the snatch. There was no significant difference between R1 and R5 based on the type of IRR treatment given, $\mathrm{F}(3,12)=$ 1.541, $\mathrm{P}<0.255$; Wilk's $\Lambda=0.722$, partial $\eta^{2}=$ .278. For KJV, the Manova test conducted showed that there was a significant difference between R1 and R5 based on the type of IRR treatment given, $\mathrm{F}(3,12)=4.144, \mathrm{P}<.031$; Wilk's $\Lambda=0.491$, partial $\eta^{2}=0.509$, indicating there is a difference between repetition one and repetition 5 of KJV between the type of IRR treatment in T1 phase. The Univariate ANOVA with Bonferroni correction tests showed a significant difference between R1 and R5 for $10 \mathrm{~s}$ IRR, $F(1,14)=6.713, P=0.021$ that there is a significant drop in terms of $\mathrm{KJV}$ in $10 \mathrm{~s}$ IRR. However, the univariate tests for 30s and 50s IRR treatment group shows no significant difference between R1 and R5, F $(1,14)=1.332, \mathrm{p}=.268, \mathrm{~F}$ $(1,14)=1.280, \mathrm{P}=0.277$ respectively. For HJV, the Manova test conducted showed no statistically significant difference between R1 and R5 based on the type of IRR treatment given, $F(3,12)=$
1.819, $\mathrm{P}<0.197 ;$ Wilk's $\Lambda=0.687$, partial $\eta^{2}=$ 0.313 .

Figure 4 demonstrates the joint velocity maintenance across repetition for the Second Pull (SP) phase. For AJV, there was a statistical significant velocity drop between R1 and R5 based on the type of IRR treatment given, F (3, $12)=6.103, \mathrm{P}<0.009$; Wilk's $\Lambda=0.396$, partial $\eta^{2}=0.604$. The univariate ANOVA with Bonferroni Correction tests further showed a significant difference between R1 and R5 for 10s IRR, $F(1,14)=10.305, P=0.006$, showing that there is a significant drop in terms of AJV in $10 \mathrm{~s}$ IRR treatment. However, the univariate tests for the 30s and 50s IRR treatment groups show no significant difference between $\mathrm{R} 1$ and $\mathrm{R} 5(\mathrm{P}=$ $0.091)$ and $(P=0.110)$. For KJV, there is no statistical significant difference between R1 and R5 based on the type of IRR treatment given, $F$ $(3,12)=2.669, \mathrm{P}<.095 ;$ Wilk's $\Lambda=0.600$, partial $\eta^{2}=0.400$. In HJV, there was no statistical significant difference across repetitions based on the type of IRR treatment given, $\mathrm{F}(3,12)=$ $1.509, \mathrm{P}<0.262$; Wilk's $\Lambda=0.726$, partial $\eta^{2}=$ 0.274 . 


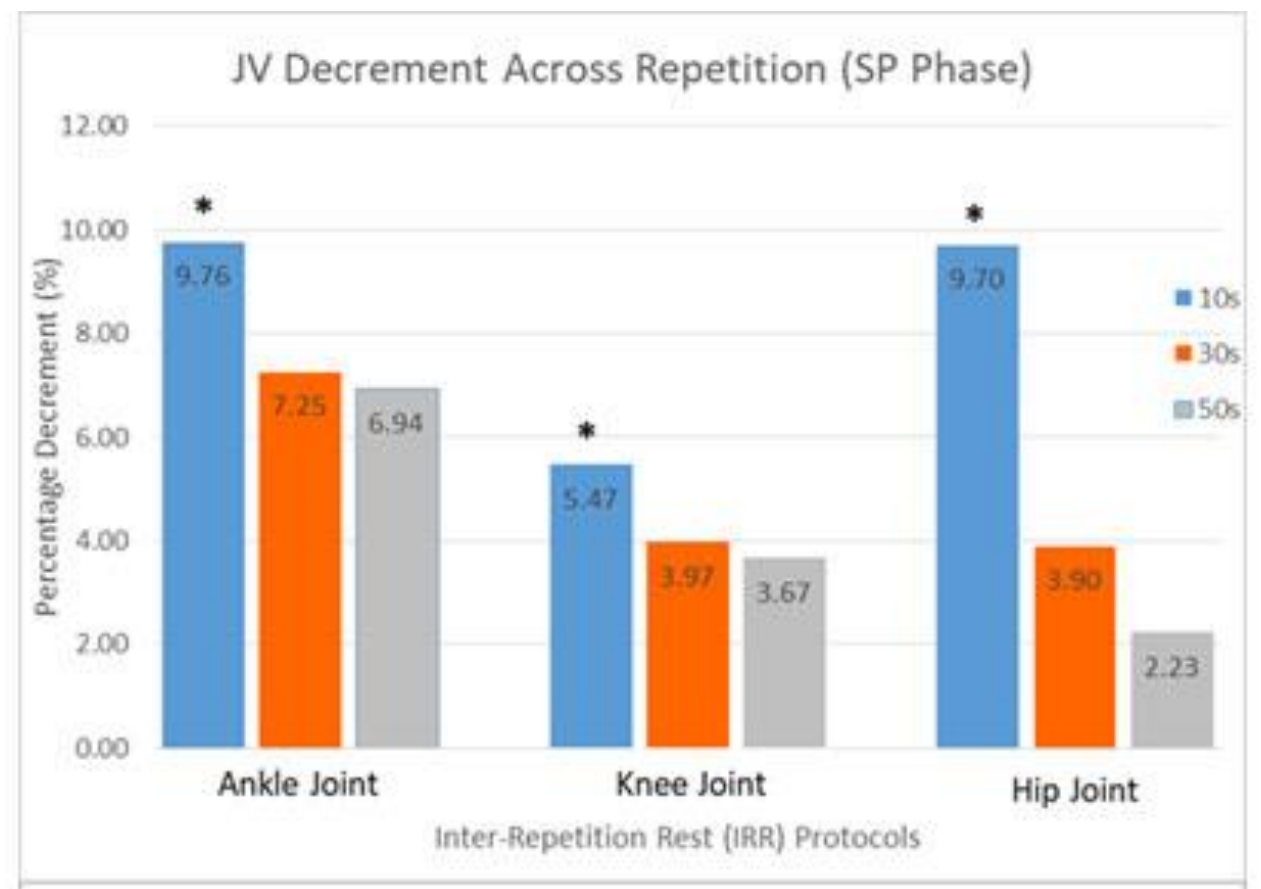

Figure 4. Jonit Velocity Decrement (\%) during Second Pull (SP) Phase Across Repetitin. * Significant drop in joint velocity during $5^{\text {th }}$ repetition $(\mathrm{P}<0.05)$.

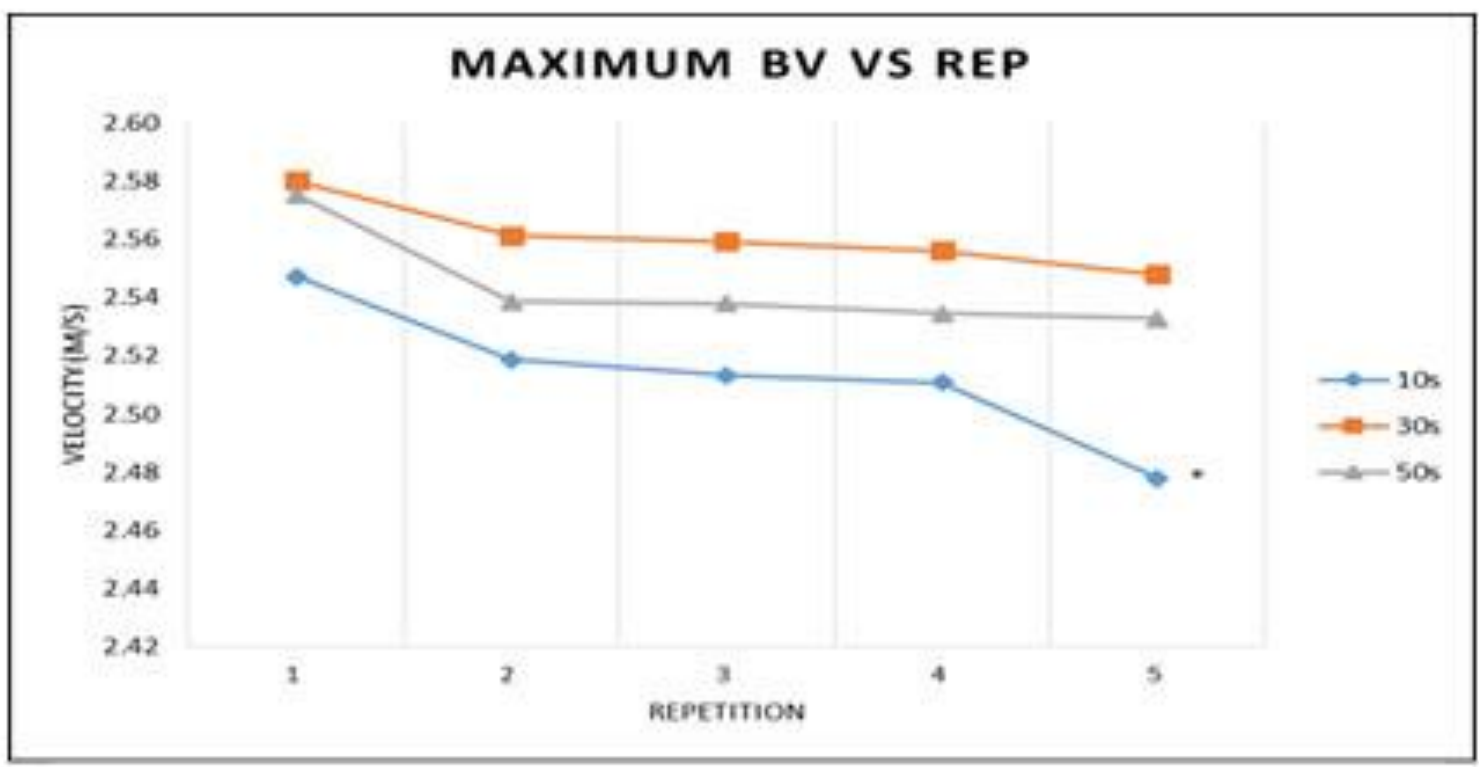

Figure 5. Maximum Barbell Velocity (m/s) Maintenance Across Repetition

Figure 5 showed the barbell velocity maintenance across repetition for all three IRR protocols (10s, 30s, and 50s). There was a statistically significant difference across repetitions based on the type of IRR treatment given, $\mathrm{F}(3,12)=4.271, \mathrm{P}=0.029$; Wilk's $\Lambda=$ 0.484 , partial $\eta^{2}=0.516$. The Univariate ANOVA with Bonferroni correction tests further showed a significant BV drop between R1 and R5 for 10s IRR, $F(1,14)=6.771, P=0.021$. However, the univariate tests for the 30 s and 50s IRR treatment groups show no significant difference between R1 and $\mathrm{R} 5(\mathrm{P}=.090$, and $(\mathrm{P}=.263)$.

\section{DISCUSSION}

Technically, the 10s IRR group displays a slow joint velocity towards all the collaborative and phases. This could indicate that fatigue is high in athletes when the 10s IRR protocol is implemented, which causes the detrimental effect of fatigue, such as velocity loss (14). As the 
repetition goes on, the muscle becomes fatigued due to lack of rest, thus slowing down the movement, affecting the joints. The 10s IRR protocol could be considered not the optimum duration to be implemented for snatch lifting training. It is insufficient and does not bring out the optimum joint velocity for a successful snatch lifting.

The first pull phase is generally slower than the second pull phase as the lifters need to lift the barbell from a stationary point. A burst in force and velocity are required during the beginning of the pull. A faster joint angle velocity would benefit the lift as it will drive into the transition phase effortlessly and transfer the force and power towards the second pull. During the transition phase, the velocity from the end of the 1 st pull should be maintained or increased (20). A loss in vertical velocity during the transition phase is deemed a technical flaw resulting in lower snatch performance $(20,21)$. From a biomechanical standpoint, the vertical barbell velocity at the end of the 2nd pull (maximum vertical acceleration) corresponds to the sum of each sub-phases impulses of the entire phase.

The 30s IRR duration can be considered as the optimum short rest duration that can be applied in between repetition based on this study as the treatment enabled a faster joint angle velocity during the FP, T1, and SP phase in all three joints observed. With increasing maximal intensity, phosphocreatine repletion was noted to replenish by $50 \%$ at 30 seconds' duration after complete depletion (22). Based on this statement, the longer the rest, the better the velocity output will be due to more significant recovery. Instead, the study discovers that the 50s IRR duration doesn't benefit the joint velocity significantly. The combined velocity output is slower when implementing the 50s IRR than the 30s IRR, although it is faster than the 10s IRR protocol. Technically this could happen because the muscle excitation decreases due to a long rest, which causes the muscle to be more relaxed compared to a shorter rest duration. They are not ready to generate an explosive burst force for the snatch exercise. Snatch is an explosive movement, so the muscle must be in a state where it is elastic and are always ready to do a sudden burst movement and giving a long rest may induce relaxation in the muscles. This could also be a physiological effect or a psychological effect that should be tested further to pinpoint the cause.
Even if the fatigue in this study did not lead to the snatch's failed lifting, it affects the joint velocity and barbell velocity, which ultimately portray how optimum the movement is. A slower rate could be associated with a higher fatigue level in an athlete. The higher the fatigue, the more energy is needed to do the same lifting repeatedly. Athletes need to lift at an optimum velocity and with less fatigue; this is possible while maintaining their performance throughout the repetition. By doing this, the athletes won't be just completing the task in a very tired and sloppy movement, which could result in injury but in a more optimum and less energy-consuming activity with little unnecessary movement causes by the negative effect of fatigue.

Overall, it can be said in this study that the $30 \mathrm{~s}$ IRR protocol seems to be the optimum duration to be implemented between the repetition rest protocols for explosive exercise such as snatch. Hardee et al. (4) showed significantly higher power, force, and velocity values with increasing IRR treatments during the power clean exercise. Lawton et al. (23) showed significant differences when implementing IRR and cluster sets compared to traditional continuous repetition bouts in the bench press exercise. This current study adds to the literature above by showing significantly higher velocity values with increasing IRR duration in the snatch exercise. However, there is a contradiction when the rest duration is more than the $30 \mathrm{~s}$ IRR treatment protocol because the data shown when implementing the 50s IRR protocol shows a lower performance output compared with the 30s IRR protocol treatment.

Although the 30s IRR treatments elicited superior performance output in joint velocity and barbell velocity, the 50s protocol velocity decrements were minimal compared to the 10 s and 30s IRR treatment group across repetitions. Specifically, the $50 \mathrm{~s}$ treatment group had decrements of joint velocity for the ankle joint $(8.17 \%, 14.04 \%$, and $6.94 \%)$, knee joint $(4.17 \%$, $12.07 \%$, and $3.67 \%)$, and hip joint $(2.04 \%, 0.95 \%$, and $2.22 \%$ ) respectively for three different phases (FP, T1, and SP) across repetition which is much lower compared to the 10s and 30s IRR protocol. The results agree with previous studies showing IRR and cluster sets to significantly affect performance maintenance in continuous repetitious bouts of exercise $(4,13,23,24)$. Lawton et al. (23) showed significant differences 
when implementing IRR and cluster sets compared to traditional continuous repetition protocol in the bench press exercise. The study showed 23 second IRR elicited a $21 \%$ higher power output than control groups with constant groups. Hardee et al. (4) showed that mean peak power dropped $14.94 \%$ throughout the set with continuous repetition bouts compared to only $5.76 \%$ decreases with 20 second IRR treatment groups.

This current study agrees with these previous studies by showing significantly higher joint velocity values, barbell velocity with increasing IRR up to 30 seconds in the snatch pull exercise. However, when using a more extended duration reaching 50s IRR, the maintenance of performance across repetition and set is better. Still, it is not the best compared to the 30s IRR treatment in terms of performance output.

Thanks to the study of the biomechanical variables above employing a 3D camera, the kinematics of the snatch techniques of weightlifters could be analyzed under a different duration of IRR implemented. Such analysis is a contribution for coaches to guide their practice in an objective and customized manner, with better skills to analyze their athletes' performance. Thus, coaches can develop more effective training programs and promote a more efficient snatch technique among their athletes, thus improving their performance.

Although an optimum IRR duration was established in this study, it would be interesting to replicate the analysis with a percentage of their personal best or weight in further studies to see if results change significantly.

\section{CONCLUSION}

Ultimately, the 30s IRR treatment is the optimum time duration suitable for inter repetition rest (IRR) protocol towards an explosive type of exercise such as snatch. However, for better maintenance of performance across repetition, the 50s IRR is the optimum time duration to be implemented. There is a meager percentage of performance drop across repeat while disregarding the performance output. Overall, 30s IRR treatment is an optimum choice for performance variables output and maintenance across repetition and sets to be recommended.

\section{APPLICABLE REMARKS}

- The ability to produce and maintain maximal joint velocity and barbell velocity during weightlifting movements are crucial for neurological and physiological adaptations. Therefore, according to this study, implementing the 30s IRR protocol while performing these movements may produce a more superior stimulus leading to an overall increase in adaptation.

- Strength coaches, athletes, researchers, and exercise practitioners can benefit alike from knowing an optimum duration of IRR protocol and the ability to implement IRR protocols to control neuromuscular fatigue and maintain a higher stimulus during weight lifting exercises, which could finally benefit during the competition for specific sports. The $30 \mathrm{~s}$ IRR protocol could be the optimum duration for IRR protocol in an explosive activity like the snatch exercise.

\section{ACKNOWLEDGEMENT}

The authors would like to express their gratitude to the UPSI Sports Biomechanics Laboratory staffs for their continuous support in this research.

\section{REFERENCES}

1. Stone MH, Pierce KC, Sands WA, Stone ME. Weightlifting: A brief overview. Strength Condition J. 2006;28(1):50. doi: 10.1519/1533-4295(2006)28[50:WABO]2.0.CO;2

2. Halil T, Nurtekin E, Serdar B, Turgut K, Ahmet S, Dede B. Effects of Fatigue on The Balance Performance as Measured by Balance Error Scoring System in Volleyball Players. Ovidius Univ Annal Series Physic Educat Sport Sci Movement Health. 2009;9(2).

3. Hardee JP, Lawrence MM, Utter AC, Triplett NT, Zwetsloot KA, McBride JM. Effect of inter-repetition rest on ratings of perceived exertion during multiple sets of the power clean. Eur J Appl Physiol. 2012;112(8):3141-3147. doi: 10.1007/s00421-011-2300-x pmid: 22215288

4. Hardee JP, Lawrence MM, Zwetsloot KA, Triplett NT, Utter AC, McBride JM. Effect of cluster set configurations on power clean technique. J Sports Sci. 2013;31(5):488-496. doi: 10.1080/02640414.2012.736633 pmid: 23121475 
5. Oliver JM, Kreutzer A, Jenke SC, Phillips MD, Mitchell JB, Jones MT. Velocity Drives Greater Power Observed During Back Squat Using Cluster Sets. J Strength Cond Res. 2016;30(1):235-243. doi: 10.1519/JSC.0000000000001023 pmid: 26121432

6. Valverde-Esteve T, Juan MG, Pablos-Monzó A, Pablos-Abella C, Juan MM, Rodríguez-Ruiz D. Effect of the inter-repetition rest length in the capacity to repeat peak power output. British J Sport Med. 2013;47(10):e3. doi: 10.1136/bjsports-2013-092558.83

7. Chiu HT, Wang $\mathrm{CH}$, Cheng KB. The three-dimensional kinematics of a barbell during the snatch of Taiwanese weightlifters. $J$ Strength Cond Res. 2010;24(6):1520-1526. doi: 10.1519/JSC.0b013e3181db23f4 pmid: 20508454

8. Ulareanu MV, Potop V, Timnea OC, Cheran C. Biomechanical Characteristics of movement phases of clean \& jerk style in Weightlifting performance. Soc Behav Sci. 2014;137:64-69. doi: 10.1016/j.sbspro.2014.05.253

9. Stone MH, Fry AC, Ritchie M, Stoessel-Ross L, Marsit JL. Injury potential and safety aspects of weightlifting movements. Strength Condition J. 1994;16(3):15-21. doi: 10.1519/10736840(1994)016<0015:IPASAO>2.3.CO;2

10. Goertzen M, Schoppe K, Lange G, Schulitz KP. [Injuries and damage caused by excess stress in body building and power lifting]. Sportverletz Sportschaden. 1989;3(1):32-36. doi: 10.1055/s-2007-993630 pmid: 2711326

11.Gonzalez-Badillo JJ, Marques MC, Sanchez-Medina L. The importance of movement velocity as a measure to control resistance training intensity. J Hum Kinet. 2011;29A:15-19. doi: 10.2478/v10078011-0053-6 pmid: 23487504

12.Haff GG, Whitley A, McCoy LB, O'Bryant HS, Kilgore JL, Haff EE, et al. Effects of different set configurations on barbell velocity and displacement during a clean pull. J Strength Cond Res. 2003;17(1):95-103. doi: 10.1519/1533-4287(2003)017<0095:eodsco>2.0.co;2 pmid: 12580663

13. Moreno SD, Brown LE, Coburn JW, Judelson DA. Effect of cluster sets on plyometric jump power. $J$ Strength Cond Res. 2014;28(9):2424-2428. doi: 10.1519/JSC.0000000000000585 pmid: 24942176

14. Sanchez-Medina L, Gonzalez-Badillo JJ. Velocity loss as an indicator of neuromuscular fatigue during resistance training. Med Sci Sports Exerc. 2011;43(9):1725-1734. doi: 10.1249/MSS.0b013e318213f880 pmid: 21311352

15. Hardee JP, Triplett NT, Utter AC, Zwetsloot KA, McBride JM. Effect of interrepetition rest on power output in the power clean. $J$ Strength Cond Res. 2012;26(4):883-889. doi: 10.1519/JSC.0b013e3182474370 pmid: 22228112

16. Baechle TR, Earle RW. Essentials of strength training and conditioning.: Human kinetics; 2008.

17. Gourgoulis V, Aggelousis N, Mavromatis G, Garas A. Three-dimensional kinematic analysis of the snatch of elite Greek weightlifters. J Sports Sci. 2000;18(8):643-652 . doi: 10.1080/02640410050082332 pmid: 10972413

18. Winter DA. Biomechanics and motor control of human movement.: John Wiley \& Sons; 2009.

19. Kipp K, Harris C. Muscle-Specific Effective Mechanical Advantage and Joint Impulse in Weightlifting. J Strength Cond Res. 2017;31(7):1905-1910. doi: 10.1519/JSC.0000000000001658 pmid: 27669187

20. Bartonietz KE. Biomechanics of the snatch: Toward a higher training efficiency. Strength Condition J. 1996;18(3):24-31. doi: 10.1519/1073-6840(1996)018<0024:BOTSTA>2.3.CO;2

21. Kipp K, Harris C. Patterns of barbell acceleration during the snatch in weightlifting competition. J Sports Sci. 2015;33(14):1467-1471. doi: 10.1080/02640414.2014.992035 pmid: 25530037

22.Maughan RJ, Maughan RJ, Gleeson M. The biochemical basis of sports performance.: Oxford University Press; 2010.

23.Lawton TW, Cronin JB, Lindsell RP. Effect of interrepetition rest intervals on weight training repetition power output. J Strength Cond Res. 2006;20(1):172-176. doi: 10.1519/R-13893.1 pmid: 16503678

24. Haff GG, Whitley A, Potteiger J. A brief review: Explosive exercises and sports performance. Strength Condition J. 2001;23(3):13-25. doi: 10.1519/00126548-200106000-00003 